\title{
Idiopathic Orbital Myositis in a 9-Year-Old Girl: A Case Report
}

\author{
Titap Yazicioglu ${ }^{1}$; Isil Kutluturk ${ }^{1, *}$ \\ ${ }^{1}$ Istanbul Kartal Dr. Lutfi Kirdar Training and Research Hospital, Eye Clinic, Istanbul, Turkey \\ ${ }^{*}$ Corresponding author: Isil Kutluturk, Istanbul Kartal Dr. Lutfi Kirdar Training and Research Hospital, Eye Clinic, Istanbul, Turkey. Tel: +90-5058611984, Fax: +90-2625266664, \\ E-mail: slkutluturk@gmail.com
}

Received: June 13, 2014; Accepted: January 4, 2015

Keywords: Myositis; Inflammatory; Orbit

\section{Dear Editor,}

Non-specific orbital inflamation (NSOI) syndrome is a non-granulomatous, non-infectious and non-neoplastic orbital disease with unknown etiology. Despite being rare in children, NSOI accounts for up to $6-17 \%$ of all pediatric orbital inflammatory diseases and several conditions such as cellulitis, rhabdomyosarcoma and leukemia are considered in the differential diagnosis (1). The most frequent subtype of NSOI is orbital myositis (29\%), followed by dacryoadenitis with myositis (8\%), orbital apex inflammation (9\%) and others (22\%) (2). It clinically presents as a mass in the eyelids, restricted ocular motility, eyelid edema and increased orbital pressure. While in adults it manifests itself more often as proptosis, it more frequently presents in children as ptosis (3). Its exact cause is unknown, however, immunologic mechanisms have been addressed (4). While a definitive diagnosis of the disease is established by means of the orbital tissue biopsies, in most cases the disease can be diagnosed by clinical and radiological examinations (5). We present a case with unusual presentation of IOIP admitted to our clinic with sudden onset of diplopia and reduction of outward gaze in the left eye.

A 9-year-old girl presented to the pediatric clinic with a nonspecific headache, eye pain and restriction of left outward gaze and diplopia. Despite systemic antibiotic therapy following a preliminary diagnosis of afebrile cellulitis, the progression of complaints were noted and our clinic was consulted. Visual acuity was 20/20 in both eyes, biomicroscopic and fundus examination was normal. The exophthalmometry readings of the right and left eye were $15 \mathrm{~mm}$ and $18 \mathrm{~mm}$, respectively. Left ocular eye movement was limited in outward gaze (Figure 1). Worth 4 dot test result was determined to be diplopia in far and near evaluation. Laboratory studies were normal.
On the orbital MRI, taken SE T1-weighted images following gadopentetate dimeglumine (Gd-DTPA) on the axial and coronal plane, left medial rectus muscle was normal but increased signal intensity on $\mathrm{T} 2 \mathrm{~W}$ sequences was noted, and the presence of marked enhancement of contrast material following intravenous administration of contrast material (IVCM) (Figure 2). The imaging of the medial rectus muscle is typical of myositis and this typical image is used for differentiating NSOI from thyroid ophthalmopathy. The systemic steroid treatment (oral prednisone $1 \mathrm{mg} / \mathrm{kg}$ tapered slowly for one month) was initiated with a preliminary diagnosis of NSOI. Diplopia and ocular motility restriction were not present a week after control examination. MRI showed regression of the isolated myositis in the medial rectus. The definitive diagnosis of orbital subacute myosistis was established.

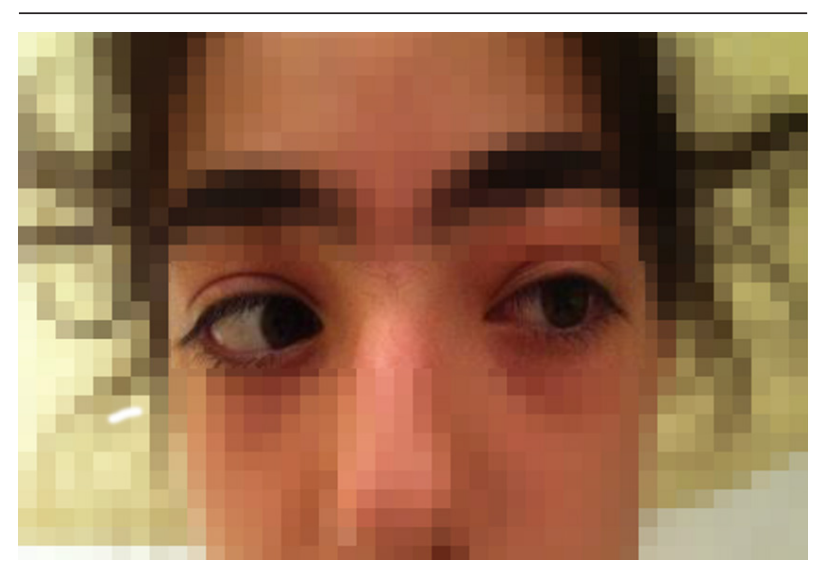

Figure 1. Limitation of Left Ocular Eye Movement 

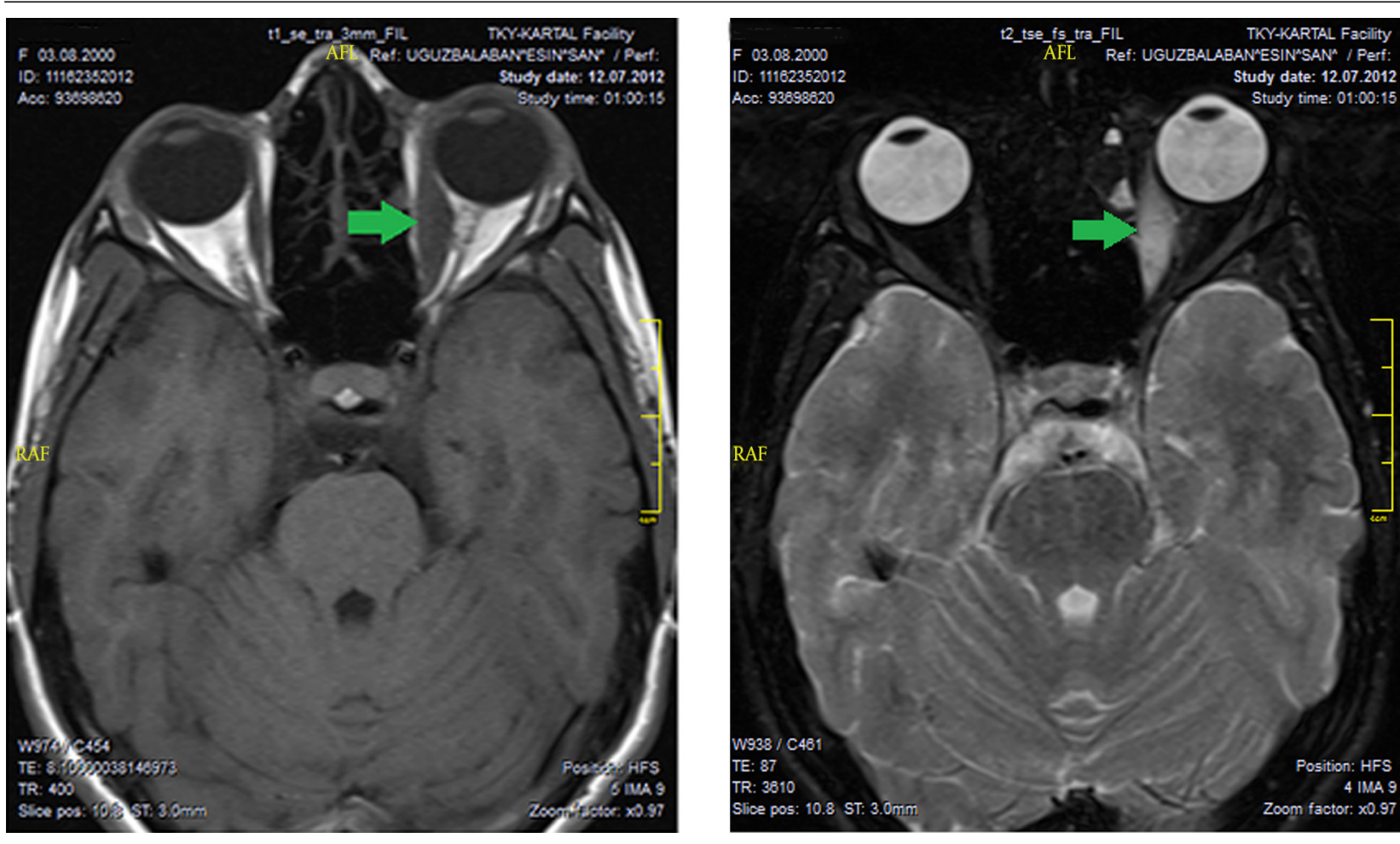

Figure 2. A, T1; B, T2

Childhood NSOI differs from NSOI in adult age. Whilst the disease seems to be one-sided in adult age, it is bilateral in childhood and recurrence rate is more common. In addition, in childhood NSOI, papilledema and iritis can be encountered $(4,5)$. Orbital myositis is a non-specific, localized inflammatory orbital process that may involve extraocular muscles. While adult and childhood NSOI cases have marked symptoms, including local orbital findings related to extraocular muscles, lacrimal gland, anterior orbit, orbital apex and diffuse inflammation, patients with orbital myositis may have only ocular motility restrictions and diplopia. In addition, these cases may present without the clinical picture proptosis and ptosis $(3,6)$. A variety of afebrile patients diagnosed with orbital cellulites go through a process of misdiagnosis not responding to antibiotic therapy (7). Moreover, some of these cases may be considered as the Graves ophthalmopathy; however, differential diagnosis is established with CT. No involvement of the tendons of the muscles occur in Graves patients, however, both muscle and tendon involvement is observed in orbital myositis cases. The acute onset of pain is more characteristic of orbital myositis. While in Graves patients the most common affected muscle is inferior rectus, in isolated orbital myositis patients the most affected muscle is medial rectus. It is important to confirm differential diagnosis with rhabdomyosarcoma as well. In rabdomyosarcoma, there exists an orbital space-occupying extremely fast growing mass, with bone erosion. Definitive diagnosis is determined by biopsy or pathological examination following excision of total mass. The inflammation associated with other orbital processes may also improve with systemic steroid administration. But with a detailed history, physical examination, directed laboratory, and radiologic studies (MR and CT), and tissue for diagnostic studies and response to anibiotic therapy may help to come to correct diagnosis. Furthermore, patients with orbital myositis respond to systemic steroid therapy more dramatically.

In conclusion, childhood orbital myositis, being usually a rarely considered diagnosis, is likely to be overlooked in childhood. Orbital myositis, a subgroup of childhood NSOI, should be included in the differential diagnosis in pediatric patients without clinically marked proptosis, ptosis, pain, fever and chemosis but with the sudden onset of ocular motility restrictions and diplopia. Thus, unnecessary use of antibiotics, unwarranted biopsies and delayed diagnoses could be avoided.

\section{References}

1. Chaudhry IA, Al-Barry MA. Pediatric orbital pseudotumor. Saudi J Ophthalmol. 2003;17(1):248-50.

2. Yuen SJ, Rubin PA. Idiopathic orbital inflammation: distribution, clinical features, and treatment outcome. Arch Ophthalmol. 2003;121(4):491-9.

3. Yan J, Qiu H, Wu Z, Li Y. Idiopathic orbital inflammatory pseudotumor in Chinese children. Orbit. 2006;25(1):1-4.

4. Mottow LS, Jakobiec FA. Idiopathic inflammatory orbital pseudotumor in childhood. I. Clinical characteristics. Arch Ophthalmol. 1978;96(8):1410-7. 


\section{Yazicioglu T et al.}

5. Mottow-Lippa L, Jakobiec FA, Smith M. Idiopathic inflammatory orbital pseudotumor in childhood. II. Results of diagnostic tests and biopsies. Ophthalmology. 1981;88(6):565-74

6. Belanger C, Zhang KS, Reddy AK, Yen MT, Yen KG. Inflammatory disorders of the orbit in childhood: a case series. Am J Ophthalmol. 2010;150(4):460-3.

7. Pollard ZF. Acute rectus muscle palsy in children as a result of orbital myositis. J Pediatr. 1996;128(2):230-3. 